\section{Dermoscopic evaluation of tinea capitis: A case report}

\author{
Ade Fernandes, Yuri Widia, \\ Sylvia Anggraeni, Linda Astari, \\ Evy Ervianti, Sunarso Suyoso \\ Department of Dermatology- \\ Venereology, Dr. Soetomo Teaching \\ Hospital / School of Medicine, \\ University Airlangga, Surabaya, \\ Indonesia
}

\begin{abstract}
Tinea capitis is the most common fungal infection in children. Recently, the dermoscopic examination of the hair and scalp or trichoscopy has surfaced on and proven to be a very effective, proficient, and efficient useful tool in diagnosing and screening of some hair disorders. Case: A 12-yearold boy presented with circular lesions with sharp margins on the scalp, with breaking hairs forming patches of partial alopecia. Scrapings of the scalp, containing broken hairs, were collected and submitted for direct examination by using $\mathrm{KOH}$ preparation revealed chains of arthroconidia covering the hair shaft. Dermoscopic examination was performed and revealed the presence of fine scale, broken hairs and comma hair. Discussion: Our patient reavealed a good clinical improvement evaluated by microscopic examination and dermoscopic evaluation. After effective antifungal therapy for 8 weeks duration, the hair fully regrows. Conclusion: Scalp dermoscopy or "trichoscopy" represents a valuable, noninvasive technique for the evaluation of patients with hair loss due to tenia capitis.
\end{abstract}

\section{Introduction}

Every year, several human and animals are infected with fungi that cause skin infections. Dermatophytes are filamentous fungi specialized on the digestion and growth on keratinized substrates, such as skin, hair, and nails. Therefore, dermatophytes are the major etiological agents of cutaneous mycoses affecting these sites. The epidemiology of dermatophytosis changes according to different geographic areas of the world, with its worldwide incidence having increased during the last decades. Moreover, they may occasionally occur in the form of outbreaks or epidemics. ${ }^{1}$

Tinea capitis is an infection of the scalp involves hyphal proliferation in the stratum corneum that extends into the hair follicle orifice and hair shaft. It affects primarily prepubertal children. This clinical form is caused by the exposure of the scalp to the inoculum from an infected individual, animal, or contaminated soil. Non inflammatory or epidemic tinea capitis may begin as a small erythematous papule around the hair shaft which spreads outward, developing fine scaling in noticeable patches. Partial or complete alopecia may result because brittle hair breaks off a few millimeters from the scalp. Affected hair may appear grey due to a coating of fungi. Noninflammatory infection is associated with M. audouinii and Microsporumferrugineum; however, T. tonsurans and $\mathrm{M}$. canis may sometimes cause noninflammatory infection. Diagnosis can be conducted easily by direct $10 \%-20 \%$ potassium hydroxide $(\mathrm{KOH})$ examination of tonsured hair, or by isolation of the dermatophyte in Sabouraud agar which would take weeks to reveal and delays diagnosis and initiation of a proper treatment. $\mathrm{KOH}$ has a high sensitivity; unfortunately, the test has a low specificity because it fails to identify the genus and species. $\mathrm{KOH}$ examination of hairs may help differentiate types of tinea capitis infection. Ectothrix infection can be distinguished from endothrix infection because in the former arthroconidia appear as chains on the surface of the hair shaft or as a mosaic sheath around the hair. Inspection under Wood's light may aid in diagnosis. Ectothrix infections with M. audouinii, M. canis, and M. ferrugineum show bright green fluorescence under the Wood's light. Cultures are essential to establish an accurate identification of the causative microorganism. Species identification is established by the macroscopic and microscopic characteristics of the culture, but it has a low sensitivity and a high specificity. Griseofulvin is the only FDA approved oral treatment; however, terbinafine, itraconazole, and fluconazole have frequently been used for the successful resolution of tinea capitis. ${ }^{2,3}$

Dermoscopy is a non-invasive diagnostic tool that allows visualization of morphologic features that cannot be seen by the naked eye. It is useful for differential diagnosis, prognostic evaluation, and monitoring the response to treatment for many cutaneous diseases. The dermoscopic examination of the hair and scalp is known as trichoscopy. Trichoscopy allows visualization of hair shafts at high magnification and performing measurements, such as hair shaft thickness, without the need of removing hair for diagnostic purposes. It also allows in vivo visualization of the epidermal portion of hair follicles and perifollicular epi-
Correspondence: Ade Fernandes, Dr. Soetomo General Hospital, Dermato-Venereology Department, Prof. Dr. Moestopo No. 47, Surabaya, East Java, Indonesia.

Tel.: +6282392982192.

E-mail: adefernandes13@gmail.com

Key words: Tineacapitis, dermoscopy, trichoscopy

Acknowledgements: The authors would like to express their genuine thanks to the Dermatovenereology Outpatient's Clinic of Dr. Soetomo General Hospital Surabaya and patient who participated in this study.

Contributions: $\mathrm{AF}$, data collecting and analyzing; $\mathrm{AF}, \mathrm{YW}$, manuscript writing; $\mathrm{AF}, \mathrm{YW}$, SA, LA, EE, SS, manuscript reviewing and references search.

Conflict of interests: the authors declare no potential conflict of interest.

Funding: None

Received for publication: 1 February 2019. Accepted for publication: 25 February 2019.

This work is licensed under a Creative Commons Attribution-NonCommercial 4.0 International License (CC BY-NC 4.0).

(C) Copyright A. Fernandes et al., 2019

Licensee PAGEPress, Italy

Dermatology Reports 2019; 11(s1):8091

doi:10.4081/dr.2019.8091

dermis. Hair and scalp disorders in children may originate from the hair itself, scalp skin, or infectious causes and may be congenital or acquired. The most common acquired causes of hair loss in children are tinea capitis and alopecia areata. The advantages of trichoscopy in evaluating hair loss in children are numerous, as it is a fast, noninvasive, inexpensive, and painless method, and therefore it will be accepted by children and their parents and is beneficial in the differential diagnosis between tinea capitis and alopecia areata in children. ${ }^{4}$

\section{Case Report}

A 12-year-old boy presented with circular lesions with sharp margins on the scalp, with breaking hairs forming patches of partial alopecia. Scrapings of the scalp, containing broken hairs, were collected and submitted for direct examination by using $\mathrm{KOH}$ preparation revealed chains of arthroconidia covering the hair shaft. Dermoscopic examination was performed and revealed the presence of fine scale, broken hairs and comma hair. Black dots, 
corkscrew hairs were not observed. At this moment, this patient was diagnosed with tinea capitis. Oral griseofulvin and ketoconazole shampoo was prescribed for this patient. After 8 days of incubation, yellow to salmon colonies started to grow on fungal culture. Subculture was performed, and the microscopic examination of the culture revealed chlamydospores, and rare fusiform macroconidia, indicating $\mathrm{M}$. audouinii as mycological agent (Figures 1 and 2).

\section{Discussion}

The clinical appearance of ringworm of the scalp is variable, depending on the type of hair invasion, the level of host resistance and the degree of inflammatory host response. Most affected patients are children for 6 months to 12 years of age. In $M$. audouinii infections, the basic lesions are patches of alopecia, often circular in shape, but Showing numerous broken-off hairs. Inflammation is minimal, but fine scaling is characteristic. ${ }^{5}$ In our case we found broken-off hairs formed patchy circular alopecia, dull grey scaling and itchy sensation.

Fluorescence under filtered ultraviolet or Wood's light is characteristically present in most ectothrix infections caused by Microsporum species. Until recently, the means of laboratory diagnosis in tinea capitis has been the combined use of direct microscopy as well as culture..$^{5}$ In our study Wood's lamp examination demonstrate bright green fluorescence of infected hairs. Samples are taken by scraping and the result revealed M. audouinii infection.

Dermoscopy is a non-invasive diagnostic technique with an already established role in the evaluation of tinea capitis and specific dermoscopic criteria have been associated with its diagnosis such as black dots, comma hairs, corkscrew hair. ${ }^{6}$ Dermoscopy is being recommended as a useful adjunctive tool in diagnosing tinea capitis. Black dot hair stubs may be visualized more clearly. 'Comma-shaped' hairs have been described in white children with ectothrix infection, whereas corkscrew hairs have been reported in Afro-Caribbean children with tinea capitis. Black dots represent cadaverised hairs, while comma hairs represent broken hairs resulting from cracking and bending of a hair shaft filled with hyphae. Corkscrew hairs represent broken coiled hairs and are considered either a variation of the commahairs in cases of naturally curly hair, or the result of a specific type of fungal parasitation. ${ }^{6,7}$ Dermoscopy of our patient revealed fine scale, broken hairs and comma hair.

Treatment for tineacapitis relies on the use of terbinafine, itraconazole, griseofulvin and fluconazole. There is no clinical evidence to support the use of other oral antifungals, including the newer azoles such as voriconazole or posaconazole.
Griseofulvin was the first effective drug used of the treatment of tinea capitis and is still widely used in resource-poor settings as it remains effective. It is useful particularly for Microsporum infections, but it is

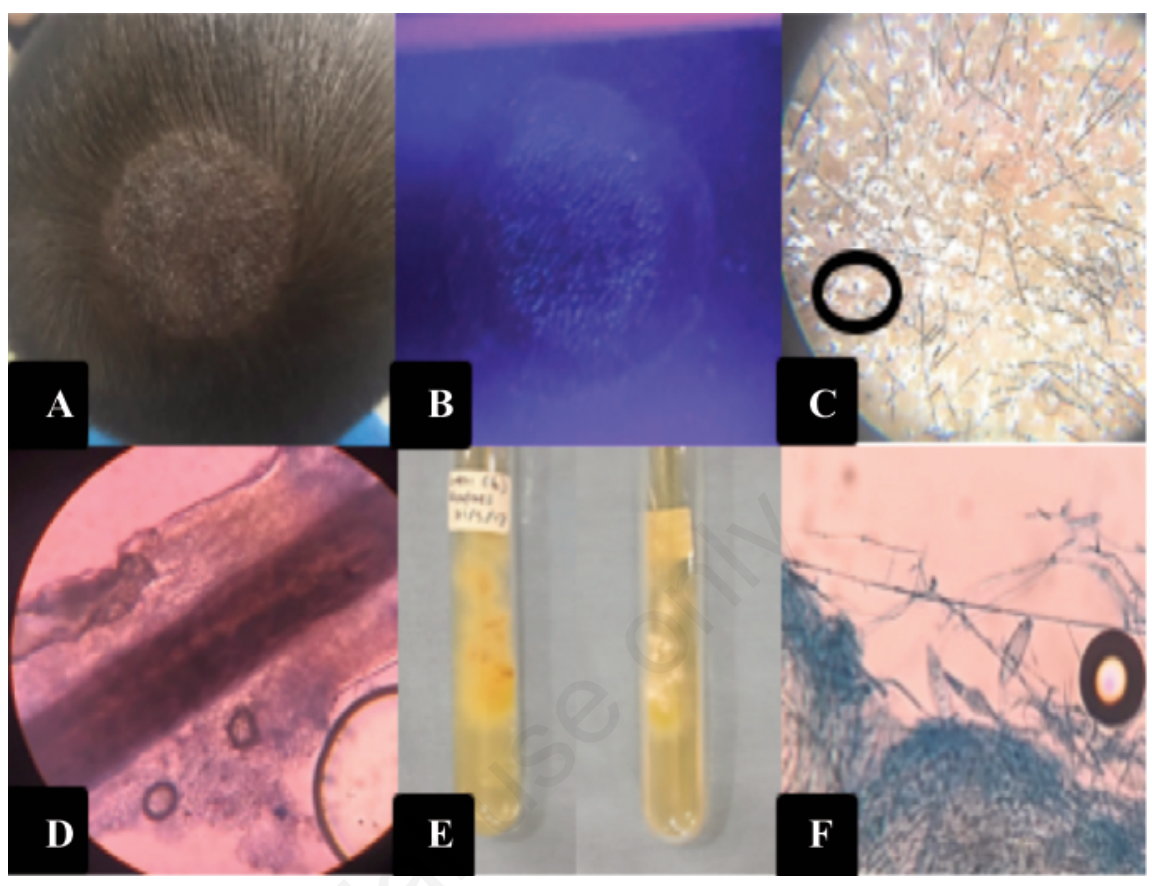

Figure 1. (A) Well-defined, round alopecia with diameter $4 \mathrm{~cm}$, grayish hair, broken hair, erythematous macule, papules, thin scale; (B) Yellow-green fluorescence on wood's light examination; (C)Dermoscopic examination before treatment revealed the presence of fine scale, broken hairs and comma hair; (D) Arthroconidiaaroundof the hair shaft (ectothrix); (E) colony morfology of flat, white and tan to salmon reverse pigment on Sabouraud agar; (F) microscopic appearance of Chlamydoconidia.

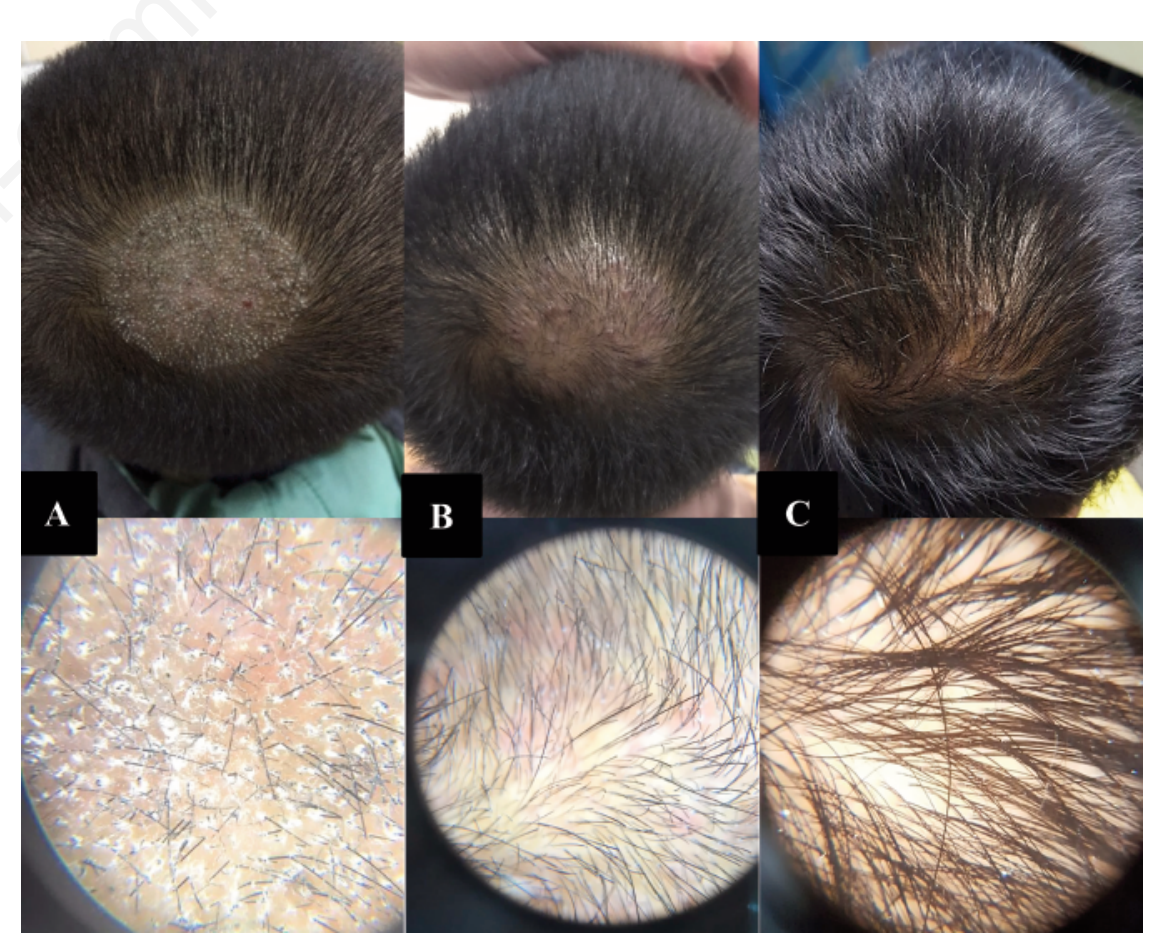

Figure 2. Clinical improvement and dermoscopic examination of tinea capitis after completed treatment for $\mathbf{8}$ weeks duration revealed the hair fully regrows. 
not available in paediatric form (liquid or small tablet sizes) in many countries. Topical antifungal therapy has little place in themanagement of tinea capitis except as an adjunct tooral therapy. There is evidence that final relapserates of infection following topical therapy are high, although the clinical appearances and itching may initially improve. ${ }^{7.8}$ Ketoconazole shampoo was used 2-3 times weekly to prevent spread in the early phases of therapy, in combination with an oral griseofulvin $20 \mathrm{mg} / \mathrm{Kg} / \mathrm{Day}$.

The definitive end point for adequate treatment must be mycological cure, rather than clinical response. Therefore, followup with repeat mycology sampling is recommended at the end of the standard treatment period and then monthly until mycological clearance is documented. Treatment should therefore be tailored to each individual patient according to response. ${ }^{7}$ Trichoscopy may be useful both for diagnosis and therapeutic monitoring of this disease. This method is simple, quick and easy to perform, is well accepted by patients and is also useful for monitoring treatment and for follow-up. ${ }^{9}$ Our patient reavealed a good clinical improvement evaluated by microscopic examination and dermoscopic evaluation. After effective antifungal therapy for 8 weeks duration, the hair fully regrows.

\section{Conclusions}

Tinea capitis is a common scalp infection, seen predominantly in childhood. The clinical presentation is highly variable and dependent on the causative organism. Tinea capitis does not respond well to topical therapy alone, thus oral therapy is requisite. The drug of choice is griseofulvin. Fungal culture should be requested in the persistent of scalp lesion. Scalp dermoscopy or "trichoscopy" represents a valuable, noninvasive technique for the evaluation of patients with hair loss due to tenia capitis. It is simple, quick, and easy to perform. This makes it a modern, non-invasive technique, which is well accepted by both, dermatologists and patients.

\section{References}

1. Amer M, Helmy A, Amer A.Trichoscopy as a useful method to differentiate tinea capitis from alopecia areata in children at Zagazig University Hospitals. Int J Dermatol 2017; 56(1): 116-120

2. Brito-Santos F, Figueiredo-Carvalho MHG, Coelho RA, Sales A, AlmeidaPaes R. Tineacapitisby Microsporumaudouinii: Case reports and review of published global literature 2000
2016. Mycopathologia 2017; 182(1112):1053-60.

3. Elewski BE. Tinea capitis: a current perspective. J AmAcadDermatol 2000;42:1-20.

4. Elghblawi E.Tinea capitis in children and trichoscopic criteria. Int $\mathrm{J}$ Trichology 2017; 9(2):47-9.

5. Fuller LC, Barton RC, MohdMustapa MF, Proudfoot LE, Punjabi SP, Higgins EM. British association of dermatologists' guidelines for the management of tinea capitis. Br J Dermatol 2014; 171(3):454-63.

6. Hay RJ. Tinea capitis: Current status. Mycopathologia 2017;182(1-2):87-93.

7. Lacarrubba F, Micali G, Tosti A. Scalp Dermoscopy or Trichoscopy. Curr Probl Dermatol 2015; 47: 21-32.

8. Lekkas D, Ioannides D, Apalla Z, Lallas A, Lazaridou E, Sotiriou E. Dermoscopy for discriminating between Trichophyton and Microsporum infections in tinea capitis. J Eur Acad Dermatol Venereol 2017; 1: 1-6.

9. Welsh O, Gonzalez GM. Dermatophytosis and Other superficial Fungal Infections. In: Hospenthal DR, Rinaldi MG, edistors. Diagnosis and treatment of fungal infection. 2nd ed. Switzerland: Springer; 2015. p.245-60. 